\title{
Effective Herding in Shepherding Problem in V-formation Control*
}

\author{
Kaoru FuJIOKA ${ }^{\dagger}$
}

\begin{abstract}
The shepherding problem is to guide or control a number of sheep by means of a small number of shepherds, which has useful applications outside the original sheep setting, such as to robotics and crowd control. Many simulations have been conducted to investigate the motion of a group, whereas less research has focused on controlling a group. Therefore, the present paper aims to clarify effective group control through the shepherding problem. Specifically, we focus on a simple algorithm called V-formation control and execute computer simulations.
\end{abstract}

\section{Introduction}

Much research has been conducted to clarify the motion of flocks, herds, and swarms. In this field, the well-known Boids model simulates the motion of birds in a flock based on three simple rules[1].

On the other hand, the shepherding problem is to guide or control a flock of sheep by means of a small number of shepherds. Techniques for the shepherding problem can be applied to not only the original sheep setting but also robotics and crowd control.

A shepherding problem in a field with obstacles is considered with simple or multiple shepherds in[2] and[4], in which shepherds move with a combined movement such as approaching the flock and turning the flock. In a circle field, a flock of ducks is controlled with a mobile robot which decides its next position from a resultant force to approach the flock and to separate from the goal[3]. In[5], a discrete model using hexagonal cells is constructed for this problem. From actual data obtained using the Global Positioning System (GPS), the motions of shepherds and sheep are analyzed in[6]. For the motion planning in the shepherding problem, an interactive method is applied in[7]. In our own previous research $[8,9]$, we introduced a simple strategy for shepherds, called $V$ formation control, in which a shepherd traces a Vshaped notch toward the flock. $\operatorname{In}[9], \mathrm{V}$-formation

\footnotetext{
* Manuscript Received Date: May 26, 2017

The material of this paper was partially presented at the 48th ISCIE International Symposium on Stochastic Systems Theory and Its Applications, which was held in November 2016, and IEEE TENCON 2016, which was held in November 2016.

† International College of Arts and Sciences, Fukuoka Women's University; 1-1-1 Kasumigaoka, Higashi-ku, Fukuoka 813-8529, JAPAN

Key Words: shepherding problem, multi-agent system.
}

control was compared with the control proposed by Strömbom in [6].

Here, we consider the shepherding problem with one or two shepherds which follow $\mathrm{V}$-formation control. The effectiveness with one shepherd is compared to that with two shepherds. Computer simulation is used to obtain angles effective for guidance in $\mathrm{V}$ formation control. Some variations of $\mathrm{V}$-formation control are also considered.

\section{Shepherding Models}

In a square field of size $250 \times 250$ meters, $N_{s}=$ 100 sheep agents (agents) are randomly distributed in the central square of size $50 \times 50$ meters, which is called the starting area (Fig. 1), at the beginning of the simulation. The $N_{d}\left(N_{d}=1,2\right)$ shepherds start to guide the agents to the target position $(125,250)$ from the start position $(125,0)$. Note that two shepherds are allowed to occupy the same position (for example, the start position).

A simulation is regarded as a success if the global center of mass (GCM) of the 100 agents (Fig. 2) is within 5 meters of the target position by 1000 steps.

We describe the behavior of agents based on previous studies as follows[6]. Each agent moves to get away from a shepherd if the shepherd is within the detection distance, $d_{\text {detect }}=65$, which is represented by a unit vector $\vec{R}_{d}$ in Fig. 3 . If two shepherds are within $d_{\text {detect }}$, then $\vec{R}_{d}$ is the unit vector of the resultant force from the two shepherds.

An agent prefers to flock together with the $n(1 \leq$ $n \leq N-1)$ nearest agents, so the agent heads toward the local center of mass (LCM) of the $n$ agents (Fig. 2 ). We refer to $n$ as the number of neighbors. The unit vector of cohesive power toward the LCM is represented by $\vec{R}_{L}$ in Fig. 3. However, if an agent approaches another agent within the repulsion distance, $d_{r}=2$, then a repulsion effect occurs. The unit vector 


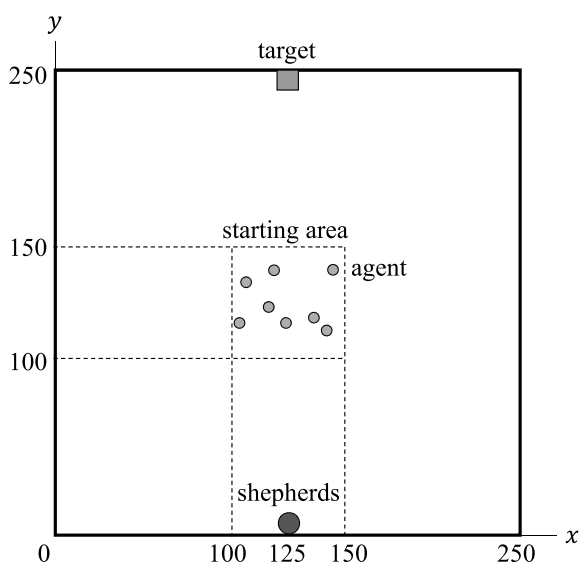

Fig. 1 Field of the shepherding model at the initial step

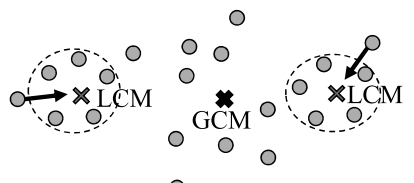

Fig. 2 Global center of mass (GCM) and local center of mass (LCM) for $n=5$

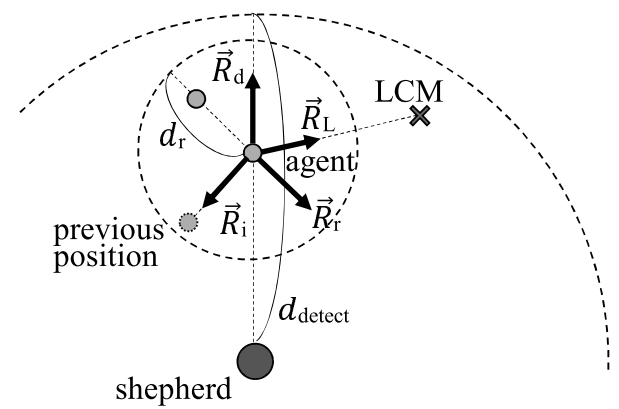

Fig. 3 Force vectors for agent

of repulsion effect is $\vec{R}_{r}$ in Fig. 3. If multiple agents are within $d_{r}$, then $\vec{R}_{r}$ is a unit vector of the resultant force from the agents.

An agent is also subject to an inertial force, which pushes the agent back to their previous position. The unit vector of inertial force is represented by $\vec{R}_{i}$ in Fig. 3. Finally, unit vector $\vec{R}_{e}$ represents a noise effect and has random direction.

Each agent decides its direction of movement using the five unit vectors as follows:

$$
\rho_{d} \vec{R}_{d}+\rho_{L} \vec{R}_{L}+\rho_{r} \vec{R}_{r}+\rho_{i} \vec{R}_{i}+\rho_{e} \vec{R}_{e} .
$$

We set $\rho_{d}=1, \rho_{L}=1.05, \rho_{r}=2, \rho_{i}=0.5, \rho_{e}=0.3$ for the parameters in (1) and the speed of the agent as 1 meter per time step based on the previous study[6], where the values are derived from the actual sheep movement collected using global positioning system (GPS).

\subsection{Shepherd Movement}

In this paper, we focus on a simple algorithm for shepherds called $V$-formation control [8,9]. In this control, a shepherd traces a V-shaped notch between the positions $P_{\text {right }}, P_{\text {center }}$, and $P_{\text {left }}$, as shown in Fig. 4, which in turn are moving toward the (fixed) target position at all times. From $P_{\text {right }}$, the shepherd next moves to positions $P_{\text {center }}, P_{\text {left }}, P_{\text {center }}$, and then $P_{\text {right }}$. If the shepherd is unable to proceed toward a next position due to the field boundary, then the shepherd skips visiting that position. The shepherd repeats this process until the simulation stops. If the distance between the GCM and the furthest agent (from the GCM) is $d_{\text {furthest }}$, then the three positions are located $d_{\text {furthest }}+d_{\text {over }}\left(d_{\text {over }}=5 d_{r}\right)$ away from the GCM. The angle between $P_{\text {right }}\left(\right.$ or $\left.P_{\text {left }}\right)$ and $P_{\text {center }}$ with respect to the GCM is $\theta(1 \leq \theta \leq 90)$ degrees. The angle $\theta$ is fixed from the start of the simulation until the end.

The speed of the shepherd is 1.5 meters per time step if there is no agent within a $3 \cdot d_{r}$ radius. Otherwise, the speed of the shepherd is set to $0.3 \cdot d_{r}$ meters per time step.

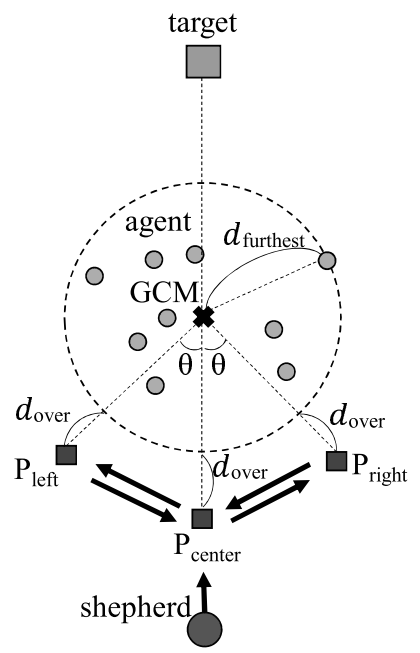

Fig. 4 Three positions and shepherd movement in Vformation control with one shepherd

Next let us consider guiding with two shepherds using V-formation control. At the beginning of the experiment, two shepherds randomly decide which part (either the left-hand side or right-hand side) of the flock to guide. For simplicity of explanation, we refer to the shepherds as left shepherd and right shepherd (Fig. 5).

Here we focus on the left shepherd. The shepherd proceeds moving between the positions $\mathrm{L}_{\mathrm{right}}$, $\mathrm{L}_{\text {center }}$, and $\mathrm{L}_{\text {left }}$. The angle between $\mathrm{L}_{\text {right }}$ (or $\mathrm{L}_{\text {left }}$ ) and $\mathrm{L}_{\text {center }}$ with respect to the GCM is $\theta(1 \leq \theta \leq 45)$ degrees. The angle $\theta$ is fixed from the start of the simulation until the end. The right shepherd moves in a similar way to the left shepherd through the positions $\mathrm{R}_{\text {left }}, \mathrm{R}_{\text {center }}$, and $\mathrm{R}_{\text {right }}$, where the angle between $\mathrm{R}_{\text {right }}$ (or $\mathrm{R}_{\text {left }}$ ) and $\mathrm{R}_{\text {center }}$ with respect to the GCM is again $\theta$ degrees. That is, the swing angles of the two shepherds are the same. We note that each shepherd independently decides its next position from the positions of agents. That is, there is no cooperation or synchronization between the two shepherds. 

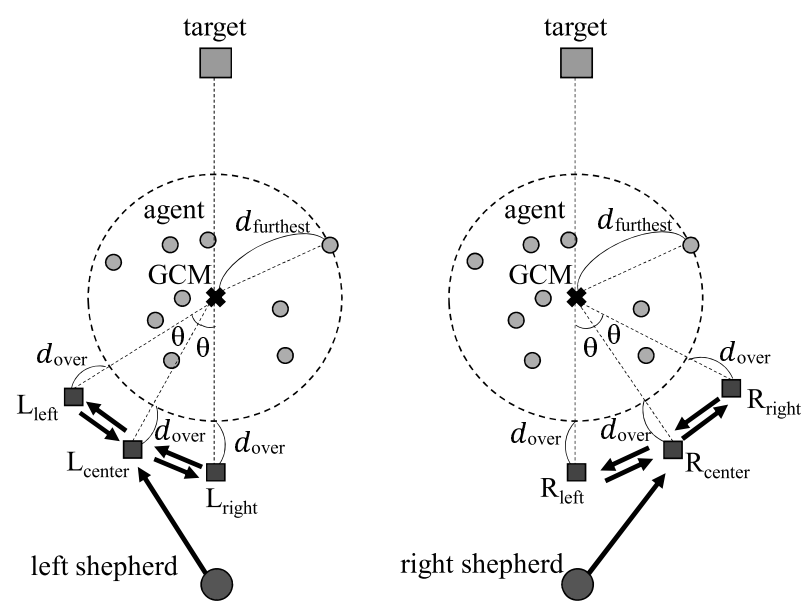

Fig. 5 Six positions in V-formation control with two shepherds

In $\mathrm{V}$-formation control, the shepherd guides the flock while "collecting" it. Based on V-formation control, we introduce the following two control algorithms with the emphasis on the collecting role. In shift $V$-formation control, a shepherd moves between the positions $P_{\mathrm{sr}}, P_{\mathrm{sc}}$, and $P_{\mathrm{sl}}$ (Fig. 6). We shift the three positions $P_{\text {right }}, P_{\text {center }}$, and $P_{\text {left }}$ in $\mathrm{V}$-formation control to $P_{\mathrm{sr}}, P_{\mathrm{sc}}$, and $P_{\mathrm{sl}}$ depending on the furthest sheep from the GCM, where the angle $\theta_{1}$ between the furthest sheep and the target position with respect to the GCM is greater than 90 degrees. Thus, no agent satisfying $\theta_{1} \leq 90$ is called the furthest agent in shift $\mathrm{V}$-formation control even if the distance between the GCM and the agent is longer than those of all other agents. The angle between $P_{\mathrm{sr}}\left(\right.$ or $\left.P_{\mathrm{sl}}\right)$ and $P_{\mathrm{sc}}$ with respect to the GCM is $\theta(1 \leq \theta \leq 90)$ degrees. The angle $\theta$ is fixed from the start of the simulation until the end.

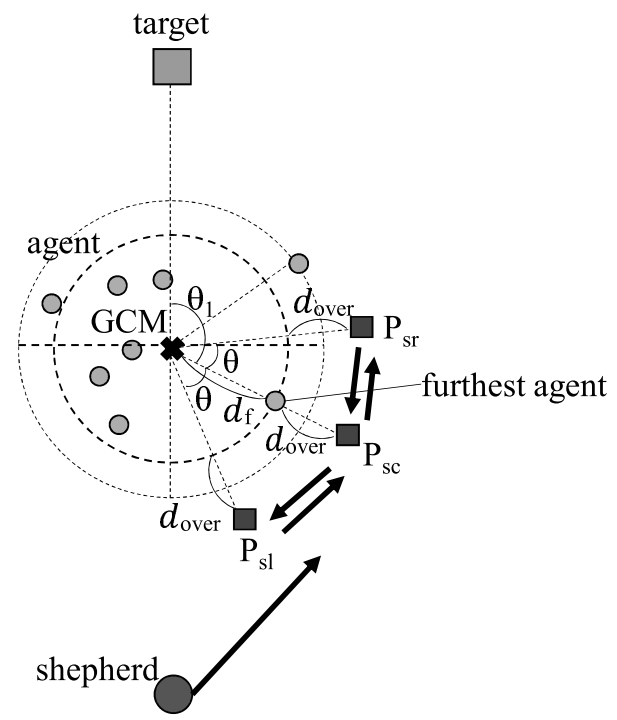

Fig. 6 Three positions and shepherd movement in shift V-formation control with one shepherd

Furthermore, we define flexible $V$-formation control, in which the shepherd moves through the posi- tions $P_{\mathrm{fr}}, P_{\mathrm{fc}}$, and $P_{\mathrm{ff}}$ (Fig. 7). The locations of these three positions are based on the furthest sheep from the GCM, where recall that the angle $\theta_{1}$ between the furthest sheep and the target position with respect to the GCM is greater than 90 degrees.

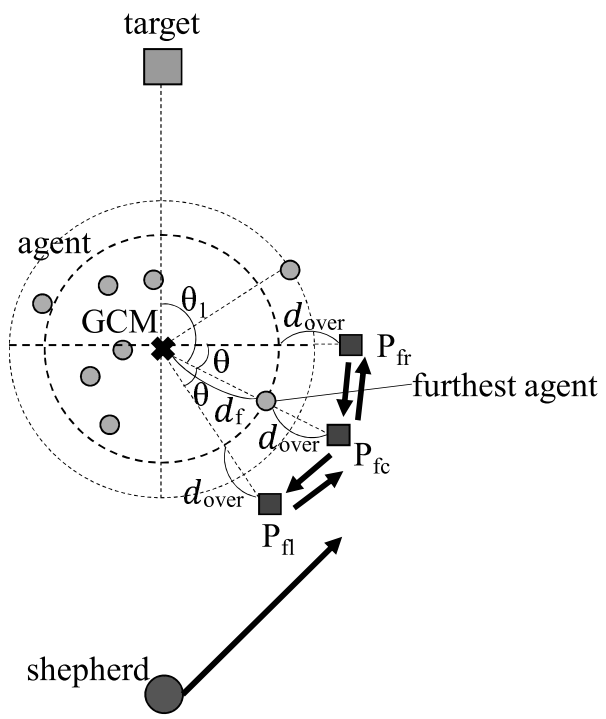

Fig. 7 Three positions and shepherd movement in flexible V-formation with one shepherd

The angle made by $P_{\mathrm{fr}}\left(\right.$ or $\left.P_{\mathrm{fl}}\right)$ and $P_{\mathrm{fc}}$, with respect to the GCM varies within an experiment. If $\theta_{1}$ satisfies $135<\theta_{1} \leq 180$, then the deflection angle $\theta$ is $180-\theta_{1}$. If $90<\theta_{1} \leq 135$, then the deflection angle $\theta$ is $\theta_{1}-90$. Therefore, the shepherd can avoid guiding the flock toward the side opposite the target position.

\section{Experimental Results}

In the following subsections, we show our results in terms of the effective angle in $\mathrm{V}$-formation control in Subsection 3.1 and the number of neighbors $n$ in Subsection 3.2.

\subsection{Effective angle}

Experiments were conducted to determine the effective angle $\theta$ in $\mathrm{V}$-formation control with one or two shepherds, as follows. In this experiment, we fix the number of neighbors $n$ and vary the angle $\theta$ from 1 to 90 (resp. 1 to 45) degrees with one shepherd (resp. two shepherds). Figs. 8, 9, and 10 show respectively the success rate, average number of steps to success, and average dispersion over 100 experiments for four numbers of neighbors, $n=10,20,30,40$. The dispersion in an experiment is defined in terms of the squares of the distances $d_{i}$ between the GCM and agents $i(1 \leq i \leq 100)$ at the end of the simulation: $\frac{d_{1}^{2}+d_{2}^{2}+\cdots+\bar{d}_{100}^{2}}{100}$. We use the dispersion to see the status of the flock at the end of the simulation because the simulation can be regarded as a success even if the flock is scattered.

From Fig. 8, the angle $\theta$ around 50 to 70 degrees for $n=30,40$ leads the flock to the target position with high probability (approximately 100 percent). 


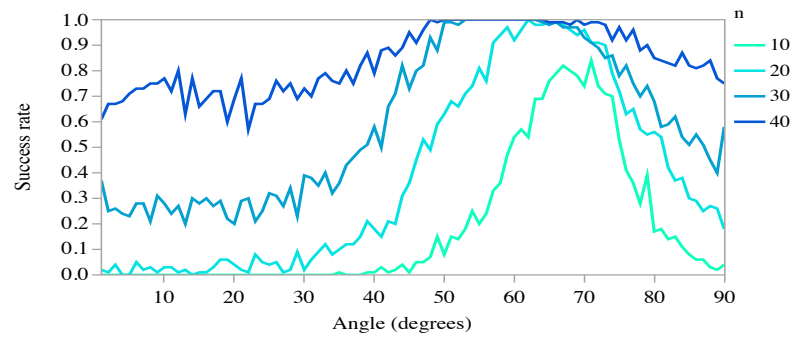

Fig. 8 Success rate in V-formation control with 1 shepherd for $n=10,20,30,40$

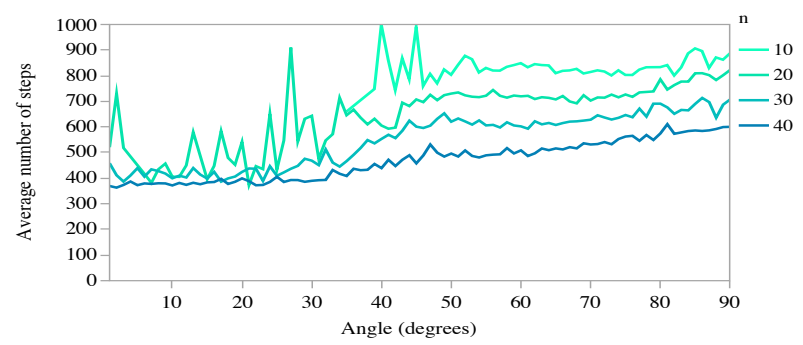

Fig. 9 Average number of steps in V-formation control with 1 shepherd for $n=10,20,30,40$

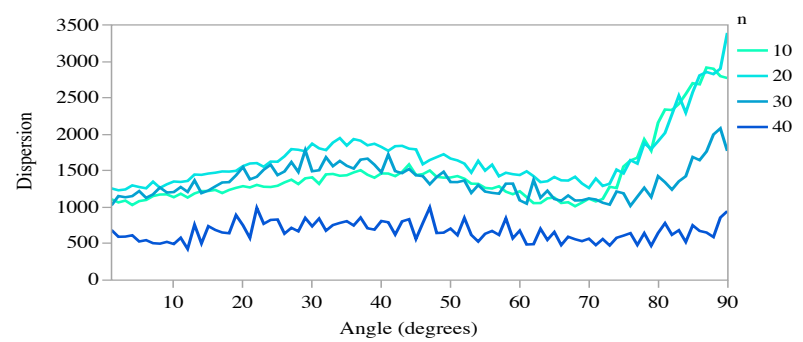

Fig. 10 Dispersion in V-formation control with 1 shepherd for $n=10,20,30,40$

For $n=10,20$, the success rate reaches its peak around 60 to 70 degrees. On the other hand, for $\theta<30$, the success rate is approximately $0.7,0.25,0.03$, and 0.0 for $n=40,30,20$, and 10, respectively. Thus it is clear from the figure that the angle has an effect on the success rate and that angles around 50 to 70 degrees are the most effective. Furthermore, a larger number of neighbors $n$ leads to a higher success rate.

For the same experiment, Fig. 9 shows the average number of steps to success. If all 100 simulations are unsuccessful, then no corresponding plot point is included. We should also consider the success rate results in Fig. 8 and ignore the results for the average number of steps for which the success rate is low. Then, generally speaking, we can expect the average number of steps to increase with an increase in angle. This is because if a larger angle is set, then the shepherd needs to take a more indirect route. However, when the angle is around 60 to 70 degrees, the average number of steps remains flat or decreases with an increase in angle especially for $n=10,20$.

For the same experiment, Fig. 10 shows average dispersion at the end of a simulation. If we ignore

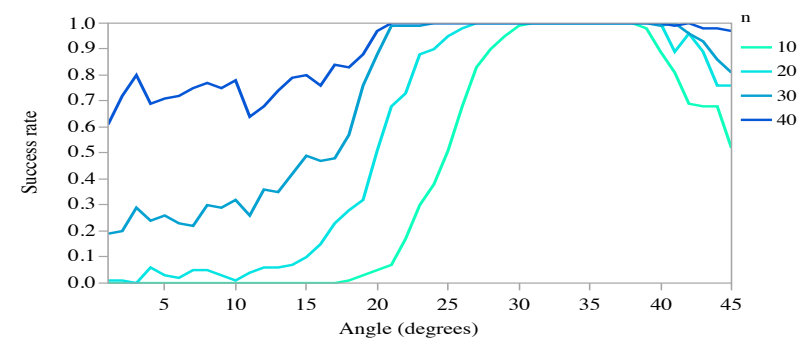

Fig. 11 Success rate in V-formation control with 2 shepherds for $n=10,20,30,40$

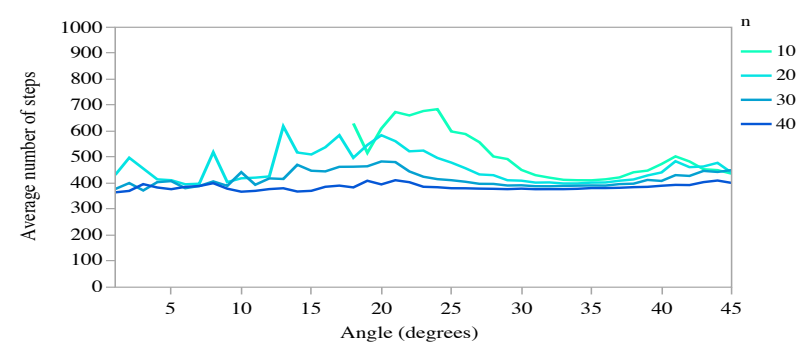

Fig. 12 Average number of steps in V-formation control with 2 shepherds for $n=10,20,30,40$

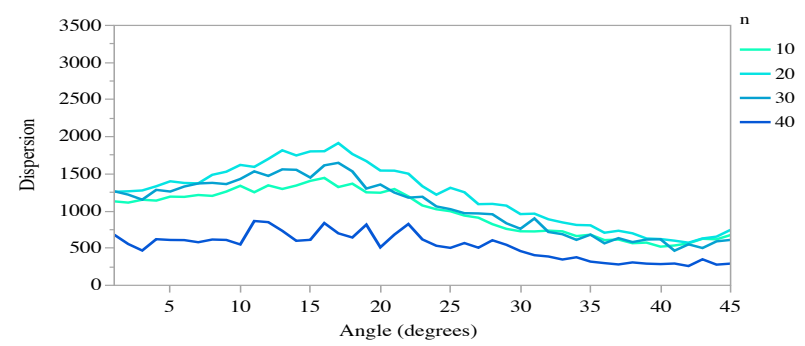

Fig. 13 Dispersion in V-formation control with 2 shepherds for $n=10,20,30,40$

the results where success rates are low (Fig. 8), the angles around 60 to 70 degrees show low dispersion regardless of $n$. Especially for $n=10,20$, the angles around 90 degrees show a higher dispersion.

From Figs. 8, 9, and 10, we can conclude that in most cases, control with a higher success rate is related to faster and more collective guidance. Taking into account success rate, speed, and dispersion comprehensively, we can conclude that an angle of $\theta$ around 60 degrees is the most effective for control.

Similarly, Figs. 11, 12, and 13 respectively show the success rate, average number of steps to success, and dispersion with two shepherds. In this experiment, we fix the number of neighbors $n(n=10,20$, $30,40)$ and vary the angle $\theta$ between 1 and 45 degrees.

For $n=10,20,30$, and 40 , the success rate is almost 100 percent for the angles around 30 to 40, 30 to 40,20 to 40 , and 20 to 40 degrees, respectively.

The results in Figs. 8 and 11 are similar, but control with two shepherds results in a higher success rate than with one shepherd, especially in the $n=10$ case.

For the same experiment, Fig. 12 shows the aver- 


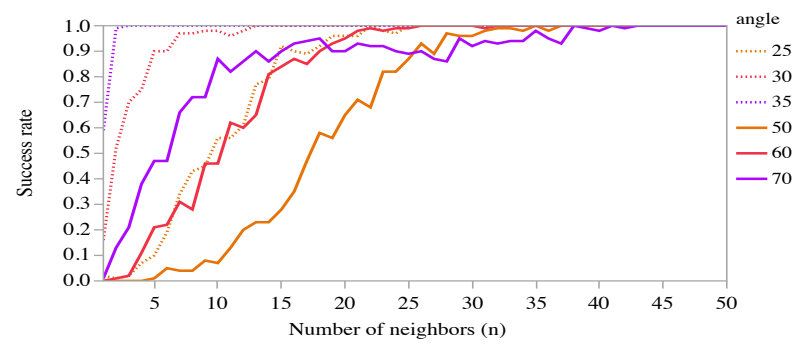

Fig. 14 Success rate in V-formation control with 1 shepherd for $\theta=50,60,70$ and 2 shepherds for $\theta=$ $25,30,35$

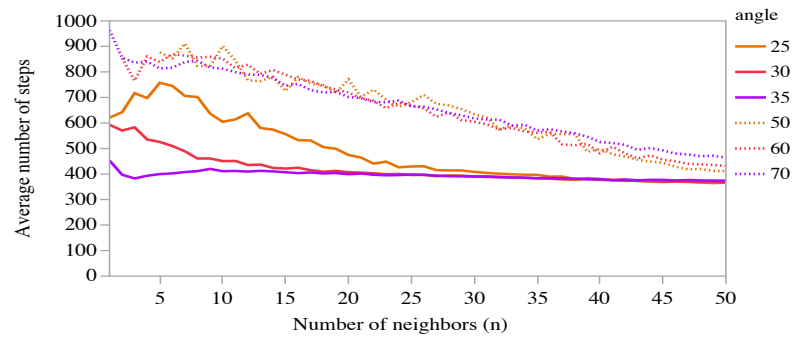

Fig. 15 Average number of steps in V-formation control with 1 shepherd for $\theta=50,60,70$ and 2 shepherds for $\theta=25,30,35$

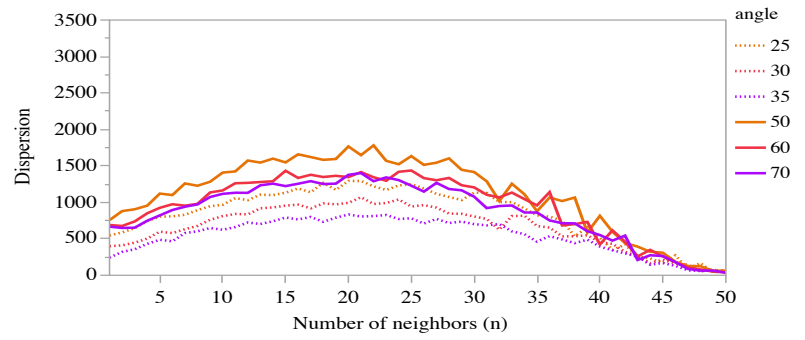

Fig. 16 Dispersion in V-formation control with 1 shepherd for $\theta=50,60,70$ and 2 shepherds for $\theta=$ $25,30,35$

age number of steps to success. The plots are fairly flat around 35 degrees at relatively small numbers of steps for all four $n$ values. Comparing Figs. 9 and 12, control with two shepherds is more stable and requires fewer steps for all $n$ values.

For the same experiment, Fig. 13 shows the dispersion at the end of the simulation. For the angles $\theta>15$, in general, the dispersion decreases with increasing angle. Comparing Figs. 10 and 13, the dispersion with two shepherds is smaller and more stable than that with one shepherd, especially for $\theta \geq 35$. In addition, the observed decreasing dispersion with increasing $\theta>15$ is not observed in the one-shepherd case (Fig. 10).

\subsection{Effects of the number of neighbors}

Next, still for V-formation control, we examine the effectiveness of two shepherds relative to one shepherd. Using the results of Subsection 3.1, we consider control for $\theta=50,60,70$ with one shepherd and $\theta=25,30,35$ with two shepherds. Fig. 14 shows the

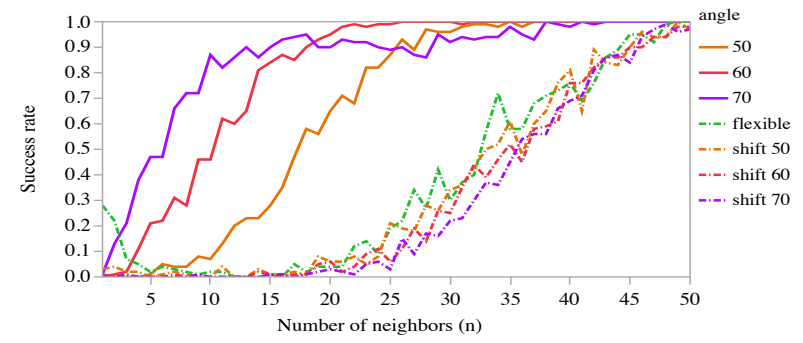

Fig. 17 Success rate in V-formation control and shift Vformation control for $\theta=50,60,70$, and flexible $\mathrm{V}$-formation control with 1 shepherd

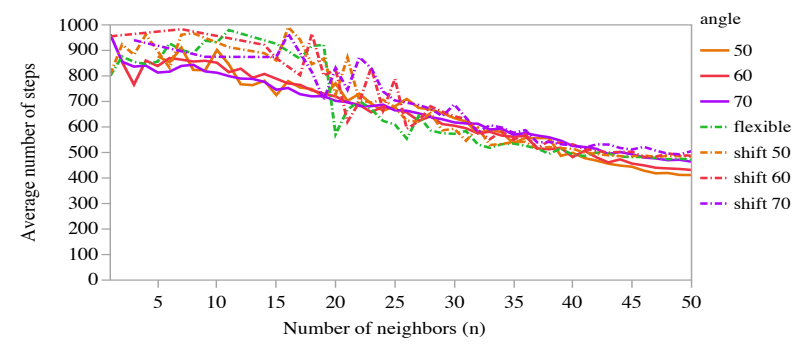

Fig. 18 Average number of steps in $\mathrm{V}$-formation control and shift V-formation control for $\theta=50,60,70$, and flexible $\mathrm{V}$-formation control with 1 shepherd

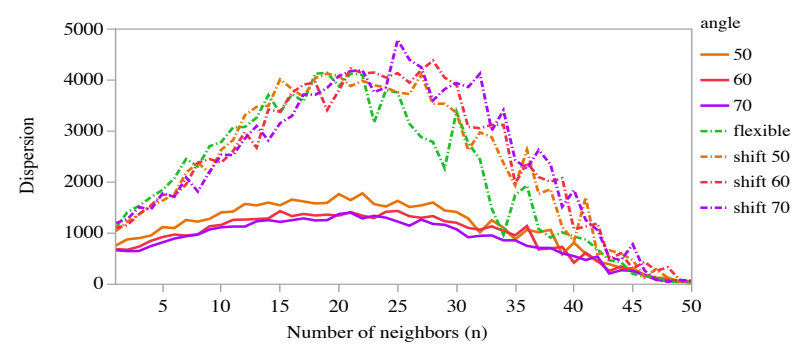

Fig. 19 Dispersion in V-formation control and shift Vformation control for $\theta=50,60,70$, and flexible V-formation control with 1 shepherd

results for success rate in $\mathrm{V}$-formation control with one shepherd and two shepherds. Comparing control for angles 25, 30, and 35 with two shepherds to control for angles 50,60, and 70 with one shepherd, the former gives higher success rates, as much as three-fold relative to $\theta=60$ with one shepherd. In fact, control for angle 35 with two shepherds gives a 100 percent success rate except for $n=1,2$.

For the same experiment, Fig. 15 shows the average number of steps. Any control with two shepherds achieves faster control than control with one shepherd.

As shown in Fig. 16, for dispersion, control with two shepherds achieves lower values than with one shepherd. From the success rate, speed, and dispersion points of view, increasing the number of shepherds gives improved results.

Next, we focus on the influence of the number of neighbors $n$. We change the value of $n$ from 1 to 50 based on the definition of the LCM. If the number of neighbors $n$ is close to 1 , agents will tend to be 
scattered, and otherwise they will form large groups. If $n$ is greater than 50 , then the flock will always move without scattering.

Fig. 17 shows the success rate in $\mathrm{V}$-formation $(\theta=50,60,70)$, shift $\mathrm{V}$-formation $(\theta=50,60,70)$, and flexible $\mathrm{V}$-formation control with one shepherd. In most cases, the success rate increases with an increasing number of neighbors. This is because if a larger $n$ is set, then agents form larger groups and control becomes easier.

Considering only $\mathrm{V}$-formation control, control with 70 degrees shows the highest success rate for $n \leq 18$, and otherwise control with 60 degrees is most successful, among $\theta=50,60,70$. For $n<25$, there is a big difference in success rate between $\theta=50$ and $\theta=70$, as much as 80 percent.

Compared with V-formation control, both shift $\mathrm{V}$-formation control and flexible $\mathrm{V}$-formation control have reduced success rates except for $n<3$ in flexible V-formation control. Flexible V-formation control shows a slightly higher success rate for $25<n<40$ than shift $\mathrm{V}$-formation control.

For the same experiment, Fig. 18 shows the average number of steps. Generally speaking, we can expect the average number of steps to decrease with an increasing number of neighbors. Flexible V-formation control has a slightly smaller success rate than the other controls for $20<n<40$, but there is no big difference between the seven controls for $n \geq 30$.

For the same experiment, the dispersion is shown in Fig. 19, where the ordinate scale is different from that of Figs. 10, 13, and 16.

For all seven controls, dispersion increases with increasing $n$ for $1 \leq n \leq 20$, then is flat for $20 \leq n \leq 25$, and decreases with increasing $n$ for $n \geq 25$. Therefore, a stronger cohesive power of agents does not always lower the post-herding dispersion. Shift Vformation control and flexible $\mathrm{V}$-formation control give higher dispersions for most values of $n$ compared with $\mathrm{V}$-formation control, while flexible $\mathrm{V}$-formation control shows lower dispersions for $n>25$ than shift V-formation control.

These results show that guiding toward the target is more effective than guiding around the furthest sheep, which rather scatters the flock.

\section{Conclusion}

In this paper based on $\mathrm{V}$-formation control, we considered control with two shepherds, and also introduced shift V-formation control and flexible Vformation control.

Any control with two shepherds shows higher success rates, fewer steps, and smaller dispersions than the corresponding control with one shepherd. Two shepherds are in particular effective even if the cohesive power is weak. It is an interesting future work to examine how many sheep agents can be controlled per shepherd.

From our experiments, V-formation control is com- prehensively more effective than shift V-formation control and flexible $\mathrm{V}$-formation control, in which a shepherd moves based on the position of the furthest agent from the center of agents called the GCM, from the points of view of success rate, speed, and dispersion. Therefore, shepherd movement based only on the center of the agents gives effective control.

In our model with two shepherds, there is no cooperation or synchronization between the two shepherds and so there is room for improvement of the shepherds' movements. Our future work is to let the two shepherds cooperate.

Our results depend on sheep agent movement and the initial setting such as the size of the field, starting area of sheep agents, the target position, the number of sheep agents, etc. Future studies are needed in order to examine our results are applicable to all other situations.

\section{Acknowledgements}

This work is supported in part by JSPS KAKENHI Grant Number 15K21308.

\section{References}

[1] C. W. Reynolds: Flocks, herds and schools: A distributed behavioral model; SIGGRAPH Comput. Graph., Vol. 21, No. 4, pp. 25-34 (1987)

[2] J. M. Lien, B. O. Bayazit, R. T. Sowell, S. Rodriguez and N. M. Amato: Shepherding behaviors; Proc. IEEE ICRA'04, pp. 4159-4164 (2004)

[3] R. Vaughan, N. Sumpter, J. Henderson, A. Frost and S. Cameron: Experiments in automatic flock control; Robotics and Autonomous Systems, Vol. 31, Nos. 1-2, pp. 109-117 (2000)

[4] J. M. Lien, S. Rodriguez, J. P. Malric and N. M. Amato: Shepherding behaviors with multiple shepherds; Proc. IEEE ICRA'05, pp. 3402-3407 (2005)

[5] Y. Sueoka, T. Kita, M. Ishikawa, Y. Sugimoto and K. Osuka: Harnessing control and performance analysis for sheepdog system: Approach from spatial discretization (in Japanese); Transactions of the JSME, Vol. 80, No. 809, pp. 1-314 (2014)

[6] D. Strömbom, R. P. Mann, A. M. Wilson, S. Hailes, J. Morton, D. J. T. Sumpter and A. J. King: Solving the shepherding problem: heuristics for herding autonomous, interacting agents; Journal of The Royal Society Interface, Vol. 11, No. 100, pp. 1-9 (2014)

[7] J. M. Lien, S. Rodriguez and E. Pratt: Interactive planning for shepherd motion; Proc. 2009 AAAI Spring Symposium, pp. 95-102 (2009)

[8] S. Hayashi and K. Fujioka: A study on the efficient flock management by multi-agent systems (in Japanese); Proc. The 78th National Convention of IPSJ, pp. 2-379-2-380 (2016)

[9] S. Hayashi and K. Fujioka: Effective shepherding behaviours using multi-agent systems; Proc. IEEE TENCON 2016, pp. 3183-3186 (2016) 


\section{Author}

Kaoru FuJIOKA (Member)

She is an Associate Professor in the International College of Arts and Sciences, Fukuoka Women's University. Her maiden name is Kaoru Onodera. She received B.S., M.S., and Ph.D degrees from Waseda University, Japan, in 2000,

2002, and 2007, respectively. Her research interests include multi-agent system, automaton, formal language theory, and DNA computing. 